\title{
Distributed Consensus of Linear Multi-Agent Systems with Switching Directed Topologies
}

\author{
Guanghui Wen $^{1}$ and Valery Ugrinovskii ${ }^{2}$
}

\begin{abstract}
This paper addresses the distributed consensus problem for a linear multi-agent system with switching directed communication topologies. By appropriately introducing a linear transformation, the consensus problem is equivalently converted to a stabilization problem for a class of switched linear systems. Some sufficient consensus conditions are then derived by using tools from the matrix theory and stability analysis of switched systems. It is proved that consensus in such a multi-agent system can be ensured if each agent is stabilizable and each possible directed topology contains a directed spanning tree. Finally, a numerical simulation is given for illustration.
\end{abstract}

\section{INTRODUCTION}

The past few years have witnessed an extensive theoretical development in the area of cooperative control of multi-agent systems. This area of control engineering has attracted significant attention from various scientific communities ranging from mathematics to electronic engineering [1], [2]. Much of this attention owes to applications in the fields of unmanned air vehicles (UAVs), smart grids, large-scale sensor networks, to name a few [2], [3], [4].

One of the central problems in cooperative control of multi-agent systems, the so called consensus problem [5], [6], is concerned with the design of local controllers using relative information to realize a state agreement in the whole group. To date, much progress has been made in the study of consensus in multi-agent systems. For example, consensus for first-order multi-agent systems with switching directed topologies has been investigated in [7], [8]. It has been shown that the state of consensus in such multi-agent systems can be achieved if and only if the time-varying network topology jointly contains a directed spanning tree. However, a large class of practical agents requires higher order dynamical models to describe dynamics of such systems [9]. Motivated by this observation, consensus problems for second-order multi-agent systems and integrator-type higher-order multiagent systems have been studied in [10], [11], [12]. More recently, the consensus problem for general linear multiagent systems has received a lot of attention [13]-[19].

This work was supported by the National Natural Science Foundation of China under Grant No. 61304168, the Natural Science Foundation of Jiangsu Province of China under Grant No. BK20130595 and in part, by the Australian Research Council through the Discovery Projects funding scheme (Project DP120102152).

${ }^{1}$ Guanghui Wen is with Department of Mathematics, Southeast University, Nanjing 210096, China. wenguanghui@gmail.com

${ }^{2} \mathrm{~V}$. Ugrinovskii is with the School of Engineering and Information Technology, The University of New South Wales Canberra at the Australian Defence Force Academy, Canberra, ACT 2600, Australia. v.ougrinovski@adfa.edu.au
Specifically, the consensus problem for linear multi-agent systems under a fixed directed communication topology has been addressed in [13], [14]. In [15], the robust consensus of linear multi-agent systems with additive perturbations of the transfer matrices of the nominal dynamics was studied. In [3] and a number of subsequent papers, the robust consensus was analyzed from the view point of the $H_{\infty}$ control theory. Among other relevant references, we mention [16] and [17] where, while assuming that the open loop systems are Lyapunov stable, the consensus problems for linear multi-agent systems with undirected and directed switching topologies have been investigated, respectively. In the situation where the multi-agent system is equipped with a leader and the topology of the system belongs to the class of switched directed topologies, the consensus tracking problem has been studied in [18], [19]. One feature of the results in these references is that the open loop agents' systems were not required to be Lyapunov stable. Note that the presence of the leader in the multi-agent systems considered in these reference facilitated the derivation and a direct analysis of the consensus error system. However, when the open loop systems are not Lyapunov stable and/or there is no designated leader in the group, the consensus problem for linear multiagent systems with switching directed topologies remains challenging.

Motivated by the above discussion, this paper aims to study the consensus problem for linear multi-agent systems with switching directed topologies. Several aspects of our study are worth mentioning. Firstly, we dispense with some of the assumptions in the existing work. E.g., the open loop dynamics of the agents are not required to be Lyapunov stable in the present paper. Furthermore, the multi-agent systems under consideration are not required to have a leader. Compared to consensus problems for linear multiagent systems that have a designated leader, the point of difference here concerns the assumption on the communication topology of the system. In the previous work on the linear leader-tracking multi-agent consensus such as, e.g., [18], each possible augmented system graph was required to contain a directed spanning tree rooted at the leader. Compared to that work, the switching topologies in the present paper are allowed to have spanning trees rooted at different nodes. This is a significant relaxation of the existing conditions since it enables the system to be reconfigured if necessary (e.g., to allow different nodes to serve as the formation leader). This also has a potential to make the system more reliable.

The analysis of leaderless tracking consensus in this paper 
proceeds as follows. By introducing a linear transformation, the consensus problem for the leaderless multi-agent system under consideration is first converted to the global uniform asymptotic stability analysis problem for a class of switched linear systems with zero equilibrium. It is worth noting that the transformation technique employed in this paper is different from those used in [20], [21] where the consensus problem was converted to the global asymptotic stability problem over a time-varying manifold. Then, by constructing multiple Lyapunov functions, sufficient conditions are derived for the system under consideration to achieve consensus. Similar to [22], our results impose a condition on the minimum dwell time of the network, i.e., the minimum time the system must spend in a particular configuration. Recall that the conditions in [22] provide a lower bound on the dwell time which ensures that consensus in a multi-agent system of coupled double integrators with switching topology is achieved if each possible topology contains a directed spanning tree. However, the analysis method given in [22] is inapplicable to general linear multi-agent systems since one can not directly calculate the divergence behavior of general linear multiagent systems.

The remainder of this paper is structured as follows. In Section II, some preliminaries from the graph theory and the problem formulation are given. In Section III, the main theoretical results are presented. A numerical example is provided in Section IV for illustration. Finally, Section V concludes the paper.

Throughout the paper, let $\mathbf{R}^{n \times n}$ and $\mathbf{N}$ be the sets of $n \times n$ real matrix space and positive natural numbers, respectively. The superscript $T$ denotes the transpose of a matrix. Unless explicitly stated otherwise, all matrices are assumed to have compatible dimensions. The matrix inequality $A>B$ means that both $A$ and $B$ are symmetric matrices and that $A-B$ is positive-definite. For a complex number $\theta, \boldsymbol{R e}(\theta)$ denotes its real part. Let $I_{n}$ be the $n$-dimensional identity matrix. The notation $\mathbf{1}_{n}$ and $\mathbf{0}_{n}$ refers to the $n$-dimensional column vectors with all entries equal to 1 and 0 , respectively. The symbols $\otimes$ and $\|\cdot\|$ denote, respectively, the Kronecker product and the Euclidean norm.

\section{Preliminaries AND Problem Formulation}

In this section, some preliminaries from the algebraic graph theory and the problem formulation are given.

\section{A. Preliminaries}

The communication topology of the considered multiagent system will be described by a switching directed graph. Let $\mathscr{G}(\mathscr{V}, \mathscr{E}, \mathscr{A})$ be a directed graph with the set of nodes $\mathscr{V}=\{1,2, \cdots, N\}$, the set of directed edges $\mathscr{E} \subseteq$ $\{(i, j), i, j \in \mathscr{V}\}$ and a weighted adjacency matrix $\mathscr{A}=$ $\left[a_{i j}\right]_{N \times N}$ with non-negative elements. The pair $(i, j)$ denotes the edge of the graph $\mathscr{G}(\mathscr{V}, \mathscr{E}, \mathscr{A})$ originating at node $j$ and ending at node $i$. The adjacency matrix $\mathscr{A}=\left[a_{i j}\right]_{N \times N}$ of a directed graph $\mathscr{G}(\mathscr{V}, \mathscr{E}, \mathscr{A})$ is defined by $a_{i i}=0$ for $i=1,2, \cdots, N$, and $a_{i j}>0$ for $(i, j) \in \mathscr{E}$ but 0 otherwise. The Laplacian matrix $\mathscr{L}=\left[l_{i j}\right]_{N \times N}$ is defined as $l_{i j}=-a_{i j}$, $i \neq j$, and $l_{i i}=\sum_{k=1}^{N} a_{i k}$ for $i=1,2, \cdots, N$. In the sequel, we will use a shorthand notation $\mathscr{G}(\mathscr{A})$ in lieu of $\mathscr{G}(\mathscr{V}, \mathscr{E}, \mathscr{A})$ if no confusion arises. A path in $\mathscr{G}(\mathscr{A})$ from node $i_{1}$ to node $i_{s}$ is a sequence of ordered edges of the form $\left(i_{k+1}, i_{k}\right)$, $k=1,2, \cdots, s-1$. A directed graph is strongly connected if and only if there exists a path between every pair of distinct nodes. A directed graph is said to contain a spanning tree if there exists a node called the root node such that there exists a directed path from this root node to every other node.

In this paper, the communication topology of the multiagent systems under consideration is assumed to be dynamically switching over a graph set $\widehat{\mathscr{G}}$, where $\widehat{\mathscr{G}}=$ $\left\{\mathscr{G}\left(\mathscr{A}^{(1)}\right), \mathscr{G}\left(\mathscr{A}^{(2)}\right), \cdots, \mathscr{G}\left(\mathscr{A}^{(p)}\right)\right\}, p \geq 1$, denotes the set of all possible directed topologies. Note that it is assumed in the present work that the graphs $\mathscr{G}\left(\mathscr{A}^{(i)}, 1 \leq i \leq p\right.$, share the same node set $\mathscr{V}$.

\section{B. Problem formulation}

Consider a multi-agent systems of $N$ agents, indexed by $1,2, \cdots, N$. The dynamics of agent $i$ are described by

$$
\dot{x}_{i}(t)=A x_{i}(t)+B u_{i}(t),
$$

where $x_{i}(t) \in \mathbf{R}^{n}$ is the state, $u_{i}(t) \in \mathbf{R}^{m}$ is the control input, $A$ and $B$ are constant real matrices with compatible dimensions. It is assumed that matrix pair $(A, B)$ is stabilizable.

Within the context of multi-agent systems, only relative information among neighboring agents can be used for coordination. For each agent $i$, the following distributed consensus protocol is proposed

$$
u_{i}(t)=\alpha K \sum_{j=1}^{N} a_{i j}(t)\left[x_{j}(t)-x_{i}(t)\right], i=1,2, \cdots, N,
$$

where $\alpha>0$ represents the coupling strength, $K \in \mathbf{R}^{m \times n}$ is the feedback gain matrix to be designed, and $\mathscr{A}(t)=$ $\left[a_{i j}(t)\right]_{N \times N}$ is the adjacency matrix of graph $\mathscr{G}(\mathscr{A}(t))$. Here, $\mathscr{G}(\mathscr{A}(t))$ describes the underlying communication topology of the agents at time $t$. Then, it follows from (1) and (2) that

$$
\dot{x}_{i}(t)=A x_{i}(t)+\alpha B K \sum_{j=1}^{N} a_{i j}(t)\left[x_{j}(t)-x_{i}(t)\right],
$$

where $i=1,2, \cdots, N$.

Definition 1: The consensus problem of multi-agent system (1) is solved by protocol (2) if, for any initial conditions, the states of the close-loop system (3) satisfy

$$
\lim _{t \rightarrow \infty}\left\|x_{i}(t)-x_{j}(t)\right\|=0, \quad \forall i, j=1,2, \cdots, N .
$$

Suppose that $\mathscr{G}(\mathscr{A}(t)) \in \widehat{\mathscr{G}}$ for all $t$. To describe the timevarying property of communication topology, assume that there exists an infinite sequence of non-overlapping time intervals $\left[t_{k}, t_{k+1}\right), k \in \mathbf{N}$, with $t_{1}=0, \tau_{1}>t_{k+1}-t_{k} \geq \tau_{0}>$ 0 , over which the communication topology is fixed. Here, $\tau_{1}>\tau_{0}>0$ and $\tau_{0}$ is called the dwell time. The introduction of the switching signal $\sigma(t):[0,+\infty) \rightarrow\{1,2, \cdots, p\}$ makes the communication topology of multi-agent system (1) well defined at every time $t$, where $t \geq 0$. For notational convenience, we will describe this communication topology using the time-varying graph $\mathscr{G}\left(\mathscr{A}^{\sigma(t)}\right)$. 
Take $x(t)=\left[x_{1}^{T}(t), x_{2}^{T}(t), \cdots, x_{N}^{T}(t)\right]^{T}$, it thus follows from (3) that

$$
\dot{x}(t)=\left[\left(I_{N} \otimes A\right)-\alpha\left(\mathscr{L}^{(\sigma(t))} \otimes B K\right)\right] x(t),
$$

where $\mathscr{L}^{(\sigma(t))}$ is the Laplacian matrix of communication topology $\mathscr{G}\left(\mathscr{A}^{(\sigma(t))}\right)$.

Before concluding this section, the following assumption is presented which will be used in the derivation of the main results.

Assumption 1: For each $i \in\{1,2, \cdots, p\}$, the graph $\mathscr{G}\left(\mathscr{A}^{(i)}\right)$ contains a directed spanning tree.

Remark 1: Note that we will not assume in the sequel that the directed spanning trees within the graphs $\mathscr{G}\left(\mathscr{A}^{(i)}\right)$, $i=1,2, \cdots, p$, share a common root node, though such an assumption is very common in the existing related literature [18], [26], [23]. Certainly, Assumption 1 holds in the special case considered in the above references, where each possible topology $\mathscr{G}\left(\mathscr{A}^{(i)}\right), i \in\{1,2, \cdots, p\}$, contains a directed spanning tree, and all these trees are rooted at the same node. Furthermore, Assumption 1 will hold if each possible topology is strongly connected.

Note that all signals considered in this paper are assumed to be differentiable on the right. Furthermore, for any given $x(0) \in \mathbf{R}^{N n}$, the switched systems (5) are assumed to have a unique and absolutely continuous solution $x(t ; x(0))$ in the sense of Carathéodory [24].

Lemma 1 ([25]): For any given $\zeta, \eta \in \mathbf{R}^{n}$, and matrices $P>0, D$ and $S$ of appropriate dimensions, one has

$$
2 \zeta^{T} D S \eta \leq \zeta^{T} D P D^{T} \zeta+\eta^{T} S^{T} P^{-1} S \eta \text {. }
$$

\section{Main Results}

In this section, the main theoretical results are presented and analyzed.

\section{A. Consensus of Linear Multi-Agent Systems with Switching} Directed Topology

Let $e(t)=\left[e_{1}^{T}(t), e_{2}^{T}(t), \cdots, e_{N-1}^{T}(t)\right]^{T}$, with $e_{i}(t)=x_{i}(t)-$ $x_{N}(t)$, for $i=1,2, \cdots, N-1$. Here, $e(t)$ represents the group disagreement vector [6]. It can then be obtained that $e(t)=$ $\left(\Xi \otimes I_{n}\right) x(t)$, where $\Xi=\left[I_{N-1},-\mathbf{1}_{N-1}\right] \in \mathbf{R}^{(N-1) \times N}$. Using this notation, it can thus be obtained from (5) that

$$
\dot{e}(t)=\left(I_{N-1} \otimes A\right) e(t)-\alpha\left(\Xi \mathscr{L}^{(\sigma(t))} \otimes B K\right) x(t) .
$$

Noticing that $\left(\mathscr{L}^{(\sigma(t))} \otimes B K\right)\left(\mathbf{1}_{N} \otimes I_{n}\right) x_{N}(t)=\mathbf{0}$, one has that

$$
\left(\Xi \mathscr{L}^{(\sigma(t))} \otimes B K\right) x(t)=\left(\Xi \mathscr{L}^{(\sigma(t))} \Pi \otimes B K\right) e(t),
$$

where $\Pi=\left[\begin{array}{c}I_{N-1} \\ \mathbf{0}_{N-1}^{T}\end{array}\right] \in \mathbf{R}^{N \times(N-1)}$. Substituting (8) into (7) gives that

$$
\dot{e}(t)=\left[I_{N-1} \otimes A-\alpha\left(\Xi \mathscr{L}^{(\sigma(t))} \Pi \otimes B K\right)\right] e(t) .
$$

Obviously, $\mathbf{0}$ is the equilibrium point of the switched system (9). Furthermore, by Definition 1 the multi-agent system (3) achieves consensus if and only if the zero equilibrium point of the switched system (9) is globally attractive. Thus, to show that the multi-agent system (3) achieves consensus it is sufficient to establish that the zero equilibrium point of the switched system (9) is globally asymptotically stable.

According to Assumption 11 it can be obtained from Theorem 2.8 in [22] that, for each $i \in\{1,2, \cdots, p\}$ and an arbitrarily given $\alpha>0$, the linear time-invariant system

$$
\dot{\zeta}(t)=-\alpha\left(\Xi \mathscr{L}^{(i)} \Pi \otimes I_{n}\right) \zeta(t)
$$

is globally asymptotically stable about its zero equilibrium point, where $\zeta(t) \in \mathbf{R}^{(N-1) n}$. This implies that for each $i \in\{1,2, \cdots, p\}$, the $(N-1) \times(N-1)$ matrix $\Xi \mathscr{L}^{(i)} \Pi$ is antistable. For notational convenience, let $\widehat{\mathscr{L}}^{(i)}=\Xi \mathscr{L}^{(i)} \Pi$, $i=1,2, \cdots, N-1$. Choose a positive scalar $c_{i}<\lambda_{\min }^{(i)}$, where $\lambda_{\min }^{(i)}=\min _{j=1,2, \cdots, N-1} \operatorname{Re}\left(\lambda_{j}\left(\widehat{\mathscr{L}}^{(i)}\right)\right)$, and $\lambda_{j}\left(\widehat{\mathscr{L}}^{(i)}\right)$, $j=1,2, \cdots, N-1$, are the eigenvalues of $\widehat{\mathscr{L}}^{(i)}$. Then it is easy to verify that there exists a positive definite matrix $Q^{(i)}$ such that

$$
\left(\widehat{\mathscr{L}}^{(i)}\right)^{T} Q^{(i)}+Q^{(i)} \widehat{\mathscr{L}}^{(i)}>2 c_{i} Q^{(i)}
$$

Remark 2: By introducing a linear transformation, the consensus problem for the multi-agent system (3) is transformed into the problem of stabilizing globally the switched linear system (9). Note that the dynamics of the group disagreement vector $e$ can be directly obtained when there is a common leader in the multi-agent systems or each possible topology is strongly connected and balanced [18], [26]. It is also worth noting that the transformation matrix $\Xi$ in 9 is not unique [27], [28].

The following theorem presents the design of a feedback gain matrix and the coupling strength for the protocol (2) to achieve consensus tracking by the closed-loop system 3 . This theorem summarizes the main theoretical results of this paper.

Theorem 1: Suppose that Assumption 1 holds and there exists a $\beta>0$ such that the following linear matrix inequality (LMI)

$$
A P+P A^{T}-B B^{T}+\beta P<0,
$$

has a feasible solution $P>0$. Then, the multi-agent system (3) with $K=(1 / 2) B^{T} P^{-1}$ achieves consensus if the following conditions hold:

(i) The coupling strength $\alpha$ satisfies the condition $\alpha>2 / c_{0}$ where $c_{0}=\min _{i=1,2, \cdots, N-1} c_{i}$, and $c_{i}, i=$ $1,2, \cdots, N-1$, are given in 111); and

(ii) For some $\kappa_{0}>0$, the switching interconnection graph $\mathscr{G}\left(\mathscr{A}^{\sigma(t)}\right)$ satisfies the following condition

$$
\beta\left(t_{k+1}-t_{k}\right)-\ln \bar{\lambda}_{\max }^{(k)}>\kappa_{0},
$$

where $\bar{\lambda}_{\max }^{(k)}$ is the largest eigenvalue of $\left(Q^{\left(\sigma\left(t_{k}\right)\right)}\right)^{-1} Q^{\left(\sigma\left(t_{k+1}\right)\right)}, k \in \mathbf{N}$.

Remark 3: It can be seen that the existence of the protocol (2) depends on the feasibility of the LMI (12). In the case where the pair $(A, B)$ is controllable, the LMI (12) is feasible for any given $\beta>0$. In the case of stabilizable but not 
completely controllable $(A, B)$, denote by $\tilde{\lambda}_{i}, i=1,2, \cdots, s$, all the uncontrollable modes of $(A, B)$. Then the LMI (12) is feasible if and only if $\beta<\min _{i=1,2, \cdots, s} \mathbf{R e}\left(-\widetilde{\lambda}_{i}\right)$.

From Theorem 1 the following corollary follows which sets a sufficient condition on the communication topology, in terms of its dwell time, for the system under consideration to achieve consensus.

Corollary 1: Suppose that Assumption 1 holds and there exists a $\beta>0$ such that the LMI (12) has a feasible solution $P>0$. Then, the multi-agent system (3) with $K=$ $(1 / 2) B^{T} P^{-1}$ achieves consensus if the coupling strength $\alpha$ satisfies the condition $\alpha>2 / c_{0}$ where $c_{0}$ was defined in Theorem 11 and the dwell time of the switching communication graph $\mathscr{G}\left(\mathscr{A}^{\sigma(t)}\right)$ satisfies the following condition

$$
\tau_{0}>\frac{\ln \bar{\lambda}_{\max }}{\beta},
$$

where $\bar{\lambda}_{\text {max }}=\max _{i, j=1,2, \cdots, p, i \neq j} \widehat{\lambda}_{i, j}$, where $\widehat{\lambda}_{i, j}$ is the largest eigenvalue of $\left(Q^{(i)}\right)^{-1} Q^{(j)}$.

Remark 4: Compared to Theorem 1 the consensus conditions given in Corollary 10 are more convenient to use in practical applications since one does not need to check the condition (13) for all time intervals. Corollary 10 tells that consensus in linear multi-agent systems with switching directed topologies with each possible topology containing a directed spanning tree can be achieved if the open-loop agent dynamics are stabilizable and the dwell time is larger than a threshold value given on the right-hand side of (14).

Remark 5: Suppose that $(A, B)$ is controllable, it can be seen from Corollary 1 that the consensus problem for the multi-agent system (3) with an arbitrarily given dwell time $\tau_{0}$ is solved by the protocol (2) designed in Theorem 1 with an appropriately selected $\beta$.

Remark 6: Since the underlying topology describing interactions between the agents is time-varying, even though the intrinsic dynamics of each agent are described by a linear time-invariant system, the close-loop agent dynamics resulting from the application of the switching protocol proposed in the present paper are indeed nonlinear. It is thus challenging or even impossible to predict the final state of consensus for such a closed-loop multi-agent system. Clearly, the final consensus value depends on the intrinsic dynamics of each agent, the coupling strength $\alpha$, the feedback gain matrix $K$, and the switching mode among different topologies.

\section{Simulation Example}

Consider a multi-agent system (3) consisting of six agents, whose topology switches between the graphs $\mathscr{G}\left(\mathscr{A}^{(1)}\right)$ and $\mathscr{G}\left(\mathscr{A}^{(2)}\right)$ shown in Figs. 1 a) and 1(b), respectively. For convenience, the weights on each edge are set to be 1 . Each agent represents a vertical take-off and landing (VTOL) aircraft. According to [29], the dynamics of the ith VTOL aircraft for a typical loading and flight condition at the air speed of $135 k t$ can be described by the system (1), with

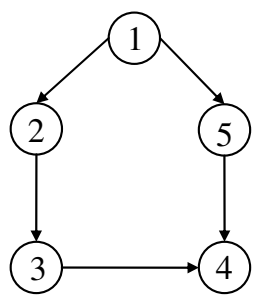

(a)

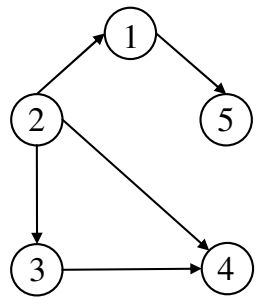

(b)
Fig. 1. Communication topologies $\mathscr{G}\left(\mathscr{A}^{(1)}\right)$ and $\mathscr{G}\left(\mathscr{A}^{(2)}\right)$.

$$
\begin{gathered}
x_{i}(t)=\left(x_{i 1}(t), x_{i 2}(t), x_{i 3}(t), x_{i 4}(t)\right)^{T} \in \mathbf{R}^{4}, \\
A=\left(\begin{array}{cccc}
-0.0366 & 0.0271 & 0.0188 & -0.4555 \\
0.0482 & -1.01 & 0.0024 & -4.0208 \\
0.1002 & 0.3681 & -0.707 & 1.420 \\
0.0 & 0.0 & 1.0 & 0.0
\end{array}\right), \\
B=\left(\begin{array}{cc}
0.4422 & 0.1761 \\
3.5446 & -7.5922 \\
-5.52 & 4.49 \\
0.0 & 0.0
\end{array}\right),
\end{gathered}
$$

where the state variables are defined as: $x_{i 1}(t)$ is the horizontal velocity, $x_{i 2}(t)$ is the vertical velocity, $x_{i 3}(t)$ is the pitch rate, and $x_{i 4}(t)$ is the pitch angle [29]. It can be seen from Fig. 1(a) that $\mathscr{G}\left(\mathscr{A}^{(1)}\right)$ contains a directed spanning tree with node 1 as the leader, while Fig. 1(b) shows that $\mathscr{G}\left(\mathscr{A}^{(2)}\right)$ contains a directed spanning tree rooted at node 2 . This reflect a hypothetical situation where the communications between aircraft 5 and 4 is unreliable, and to compensate for this, the network switches between the two topologies, with aircraft 2 assuming the role of the formation leader during the time intervals when it supplies information to agent 4 .

The transformed Laplacian matrices $\widehat{\mathscr{L}}^{(1)}, \widehat{\mathscr{L}}^{(2)}$ in this example are

$$
\widehat{\mathscr{L}}^{(1)}=\left(\begin{array}{cccc}
1 & 0 & 0 & 0 \\
0 & 1 & 0 & 0 \\
1 & -1 & 1 & 0 \\
1 & 0 & -1 & 2
\end{array}\right), \quad \widehat{\mathscr{L}}^{(2)}=\left(\begin{array}{cccc}
2 & -1 & 0 & 0 \\
1 & 0 & 0 & 0 \\
1 & -1 & 1 & 0 \\
1 & -1 & -1 & 2
\end{array}\right) .
$$

Set $c_{1}=c_{2}=0.25$. Solving the LMI (11) gives that $\bar{\lambda}_{\max }=$ 3.3225 , where $\bar{\lambda}_{\text {max }}$ is defined in Corollary 1, Let $\beta=3$, solving LMI (12) gives that

$$
K=\left(\begin{array}{cccc}
5.8206 & 0.2978 & -0.2615 & -2.7967 \\
-1.1646 & -0.4522 & 0.0530 & 2.0420
\end{array}\right) .
$$

Set $\alpha=8.1>2 / c_{0}=8.0$. Then, according to Corollary 1, one knows that consensus in the closed-loop multi-agent system (3) can be achieved if the dwell time $\tau_{0}>0.4002$. In simulations, let the topology switches among graph $\mathscr{G}\left(\mathscr{A}^{(1)}\right)$ and $\mathscr{G}\left(\mathscr{A}^{(2)}\right)$ every 0.5 second. The state trajectories of the closed-loop multi-agent system (3) are shown in Figs. 2 , 3 . The evolution of $\|e(t)\|$ is shown in Fig. 4 which confirms that the multi-agent system (3) achieves consensus. 

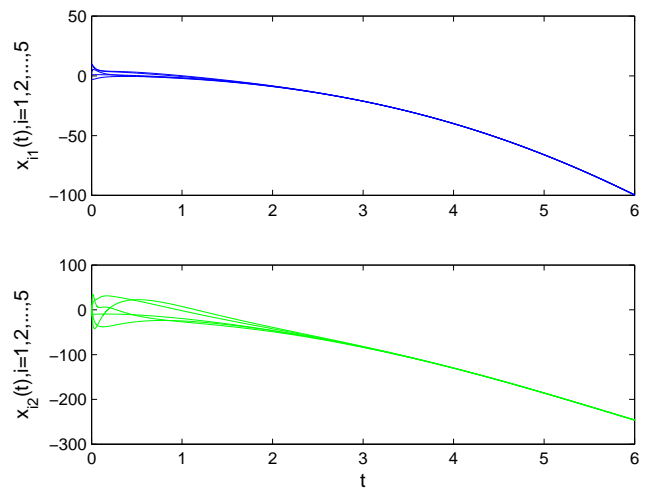

Fig. 2. State trajectories $x_{i 1}(t)$ and $x_{i 2}(t), i=1,2, \cdots, 5$.
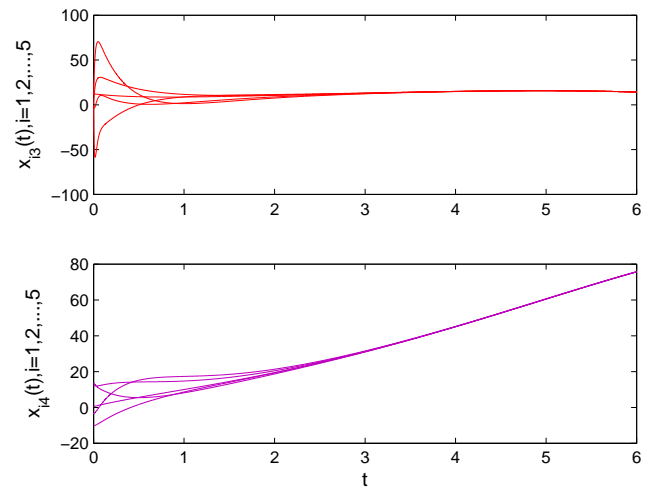

Fig. 3. State trajectories $x_{i 3}(t)$ and $x_{i 4}(t), i=1,2, \cdots, 5$.

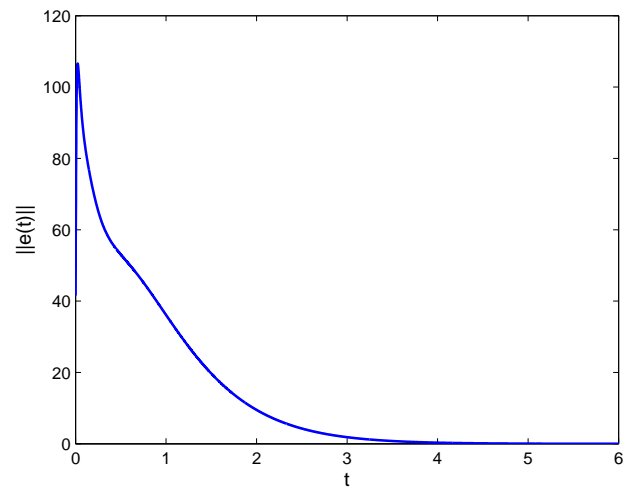

Fig. 4. Evolution of $\|e(t)\|$.

\section{CONCLUSIONS}

In this paper, distributed consensus of linear multi-agent systems has been studied where the underlying topology of agent interconnections is assumed to be time-varying. The commonly used assumption in the existing literature that the open loop agent dynamics are Lyapunov stable has been removed in the present work. Under a mild condition that each possible topology only contains a directed spanning tree and the open loop agent dynamics are stabilizable, sufficient conditions for consensus, in terms of the graph dwell time, have been derived and discussed. Future work will focus on solving the distributed consensus of linear or nonlinear multiagent systems with switching directed topologies where some of the possible topologies do not contain a directed spanning tree.

\section{REFERENCES}

[1] R. Olfati-Saber, J. A. Fax, and R. M. Murray, Consensus and cooperation in networked multi-agent systems, Proc. IEEE, vol. 95, no. 1, pp. 215-233, 2007.

[2] W. Ren, R. W. Beard, and E. Atkins, Information consensus in multivehicle cooperative control: Collective group behavior through local interaction, IEEE Control Systems Magazine, vol. 27, no. 2, pp. 71-82, 2007.

[3] V. Ugrinovskii, Distributed robust filtering with $H_{\infty}$ consensus of estimates, Automatica, vol. 47, no. 1, pp. 1-13, 2011.

[4] S. Mei, X. Zhang, and M. Cao. Power Grid Complexity. SpringerVerlage: Berlin. 2011.

[5] A. Jadbabaie, J. Lin, and A.S. Morse, Coordination of groups of mobile autonomous agents using nearest neighbor rules, IEEE Transactions on Automatic Control, vol. 48, no. 6, pp. 988-1001, 2003.

[6] R. Olfati-Saber and R. M. Murray, Consensus problems in networks of agents with switching topology and time-delays, IEEE Transactions on Automatic Control, vol. 49, no. 9, pp. 988-1001, 2004.

[7] W. Ren and R. W. Beard, Consensus seeking in multiagent systems under dynamically changing interaction topologies, IEEE Transactions on Automatic Control, vol. 50, no. 5, pp. 655-661, 2005.

[8] L. Moreau, Stability of multiagent systems with time-dependent communication links, IEEE Transactions on Automatic Control, vol. 50, no. 2, pp. 169-182, 2005.

[9] W. Ren, On constrained nonlinear tracking control of a small fixedwing UAV, Journal of Intelligent and Robotic Systems, vol. 48, no. 4, pp. 525-537, 2007.

[10] W. Ren and E. Atkins, Distributed multi-vehicle coordinated control via local information exchange, Int. J. Robust and Nonlinear Control, vol. 17, nos. 10-11, pp. 1002-1033, 2007.

[11] G. Wen, Z. Duan, W. Yu, and G. Chen, Consensus in multi-agent systems with communication constraints, Int. J. Robust and Nonlinear Control, vol. 22, no. 2, pp. 170-182, 2012.

[12] P. Wieland, J. Kim, and F. Allgöwer, On topology and dynamics of consensus among linear high-order agents, International Journal of Systems Science, vol. 42, no. 10, pp. 1831-1842, 2011.

[13] Z. Li, Z. Duan, G. Chen, and L. Huang, Consensus of multiagent systems and synchronization of complex networks: a unified viewpoint, IEEE Transactions on Circuits Systems I: Regular Papers, vol. 57, no. 1, pp. 213-224, 2010

[14] H. Zhang, F. L. Lewis, and A. Das, Optimal design for synchronization of cooperative systems: State feedback, observer and output feedback, IEEE Transactions on Automatic Control, vol. 56, no. 8, pp. 14981952, 2011

[15] H. L. Trentelman, K. Takaba, and N. Monshizadeh, Robust synchronization of uncertain linear multi-agent systems, IEEE Transactions on Automatic Control, vol. 58, no. 6, pp. 1511-1523, 2013.

[16] Y. Su and J. Huang Stability of a class of linear switching systems with applications to two consensus problems, IEEE Transactions on Automatic Control, vol. 57, no. 6, pp. 1420-1430, 2012.

[17] J. Wu, J. Qin, C. Yu, and F. Allgöwer, Leaderless synchronization of linear multi-agent systems under directed switching topologies: An invariance approach, Proc. 52nd IEEE Conf. Decision and Control, pp. 4043-6048, 2013.

[18] G. Wen, G. Hu, W. Yu, J. Cao, and G. Chen, Consensus tracking for higher-order multi-agent systems with switching directed topologies and occasionally missing control inputs, Systems \& Control Letters, vol. 62, no. 12, pp. 1151-1158, 2013.

[19] G. Wen, Z. Duan, W. Ren, and G. Chen, Distributed consensus of multi-agent systems with general linear node dynamics and intermittent communications, Int. J. Robust and Nonlinear Control, doi: 10.1002/rnc.3001.

[20] M. Huang and J. H. Manton, Stochastic consensus seeking with noisy and directed inter-agent communication: Fixed and randomly varying topologies, IEEE Transactions on Automatic Control, vol. 55, no. 1, pp. 235-241, 2010. 
[21] W. Yu, G. Chen, M. Cao, and J. Kurths, Second-order consensus for multiagent systems with directed topologies and nonlinear dynamics, IEEE Transactions on Systems, Man, and Cybernetics, Part B: Cybernetics, vol. 40, no. 3, pp. 881-891, 2010.

[22] W. Ren and R. W. Beard. Distributed Consensus in Multi-vehicle Cooperative Control: Theory and Applications. Springer-Verlag, London, 2008.

[23] L. Gao, X. Zhu, W. Chen, and H. Zhang, Leader-following consensus of linear multiagent systems with state observer under switching topologies, Mathematical Problems in Engineering, vol. 2013, Article ID 873140, pp. 1-12, 2013.

[24] D. Liberzon, Switching in Systems and Control. Birkhäuser, Boston, MA, 2003.

[25] R. A. Horn, C. R. Johnson. Matrix Analysis. Cambridge University Press, Cambridge, 1990.

[26] G. Wen, W. Yu, J. Cao, G. Hu, and G. Chen, Consensus control of switching directed networks with general linear node dynamics, Proc. 9th Asian Control Conference, pp. 1-6, 2013.

[27] W. Yu, J. Cao, and J. Lü, Global synchronization of linearly hybrid coupled networks with time-varying delay, SIAM J. Applied Dynamical Systems, vol. 7, no. 1, pp. 108-133, 2008.

[28] Y. Sun and L. Wang, Consensus of multi-agent systems in directed networks with nonuniform time-varying delays, IEEE Transactions on Automatic Control, vol. 54, no. 7, pp. 1607-1613, 2009.

[29] K. Narendra and S. Tripathi, Identification and optimization of aircraft dynamics, Journal of Aircraft, vol. 10, no. 4, pp. 193-199, 1973. 